\title{
Collaborative care depression management for older adults: level of comorbidity does not affect outcome
}

Harpole LH, Williams JW, Olsen MK, et al. Improving depression outcomes in older adults with comorbid medical illness. Gen Hosp Psychiatry 2005;27:4-12.

Q Does comorbid medical illness modulate the effectiveness of collaborative care management for depression in older adults?

METHODS

Design: Randomised controlled trial.

2.

Allocation: Not clear.

Blinding: Single blind (assessors blind to study assignment)

Follow up period: Twelve months.

Setting: Eighteen primary care clinics in the United States; time period not stated.

Patients: 1801 people aged over 60 years old, with current major depression or dysthymia (DSM-IV). Each participant's number of chronic medical comorbidities diagnosed or treated over the previous three years was ascertained. Exclusion criteria included: history of bipolar disorder or psychosis, current treatment by a psychiatrist, current drinking problems or severe cognitive impairment, or acute risk of suicide.

D Intervention: Improving Mood: Providing Access to Collaborative Treatment (IMPACT) intervention or usual care. Participants receiving IMPACT collaborative care management were assigned a depression clinical specialist (DCS; either a nurse or psychologist) who provided participant education and worked with the participant and healthcare professionals (the participant's physician, a liaison primary care expert, and a psychiatrist) to plan and monitor the participant's care. Treatment followed a stepped care algorithm and could include antidepressants, or psychotherapy provided by the DCS (6-8 sessions of problem solving treatment). The participant had weekly or biweekly contact with the DCS during acute treatment, and monthly thereafter.

Outcomes: Severity of depression (Symptom Checklist-90 (SCL$20)$ score); remission of depression ( $\mathrm{SCL}-20$ score $<0.5$ ), treatment response ( $\geqslant 50 \%$ decrease in SCL-20 from baseline), and functional status (Mental Component Score of the Short Form 12). Differences between the intervention and usual care were calculated for all participants, and also for the subpopulation with two comorbidities (the first quartile) and the subpopulation with five comorbidities (the third quartile). To assess the effect of comorbidity on treatment effect, between group treatment differences were compared for these two subpopulations.

Patient follow up: $83 \%$ at 12 months' follow up.

For correspondence: Linda Harpole, MD MPH, Department of Medicine Duke University Medical Center, Durham, NC 27709, USA; Linda.h. harpole@gsk.com

Sources of funding: John A Hartford Foundation, California Healthcare Foundation, Hogg Foundation, Robert Wood Johnson Foundation.

\section{MAIN RESULTS}

Overall, collaborative care management improved depressive symptoms $(\mathrm{p}<0.001)$, functional status, and remission and response rates compared with usual care over 12 months. Participants' number of comorbidities had no effect on the efficacy of the intervention at 12 months (comparison of between group differences in subpopulation with two comorbidities $v$ subpopulation with five comorbidities: depressive symptoms $\mathrm{p}=0.45$; remission rate $\mathrm{p}=0.29$; response rate $\mathrm{p}=0.46$; functional status $\mathrm{p}=0.83$ ).

\section{CONCLUSIONS}

The level of comorbidity in depressed older adults does not modulate the effectiveness of collaborative care management.

\section{Commentary}

7 he relation between comorbid medical illness and depression is complex. Some studies have suggested that treatment for depression may be less effective in people with comorbid medical illness, ${ }^{2} 3$ and many antidepressant trials explicitly exclude people with significant medical and psychiatric comorbidity.

Harpole et al present a subanalysis of the Improving Mood-Promoting Access to Collaborative Treatment (IMPACT) study of collaborative care management in elderly adults with depression. Participants randomised to collaborative care management had access for up to 12 months to a depression care manager (a nurse or psychologist, supervised by a psychiatrist) who provided education, care management, and brief psychotherapy (problem solving) or support of antidepressant treatment. Surprisingly, although people with more chronic medical conditions had higher depression severity at baseline, the number of chronic diseases did not affect the likelihood of response to the intervention when compared with usual care. Those receiving the intervention experienced significantly lower depression at all follow up time points compared with people receiving usual care, independent of the level of medical comorbidity.

This study's findings are important because many elderly adults with depression also have chronic medical conditions, and the study should provide much needed encouragement for clinicians treating depression in these patients. Future research should consider strategies that will optimise outcomes for this very important population.

David K Conn MB FRCPC University of Toronto, Toronto, Ontario, Canada

1 Brown C, Schulberg HC. Diagnosis and treatment of depression in primary medical care practice: the application of research findings to clinical practice. J Clin Psychol 1998;54:303-14.

2 Cole MG, Bellevance F, Mansour A. Prognosis of depression in elderly community and primary care populations: a systematic review and metaanalysis. Am J Psychiatry 1999;156:1182-9.

3 Poplin MK, Callies AL, Mackenzie TB. The outcome of antidepressant use in the medically ill. Arch Gen Psychiatry 1985;42:1 160-3. 